International Journal of Information Acquisition

Vol. 4, No. 4 (2007) 377-379

(C) World Scientific Publishing Company

\title{
AUTHOR INDEX Volume 4 (2007)
}

Althoefer, K., see Song, X.

Amaro-Camargo, E., Reyes-García, C. A., Arch-Tirado, E. \& Mandujano-Valdés, M., Statistical Vectors of Acoustic Features for the Automatic Classification of Infant Cry

Arch-Tirado, E., see

Amaro-Camargo, E.

Cai, H., see Xu, Y.

Chen, H., see Yin, X.-L.

Chen, M., Huang, B. \& Xu, Y., Abnormal Gait Detection Using Intelligent Shoes

Chen, M., see Huang, B.

Chen, W., Liang, H., Mei, T., You, Z., Miao, S., Li, S., Zhou, Y. \& Meng, M. Q.-H., Design and Implementation of Wireless Sensor Network for Robot Navigation

Chen, Y.-T., see Pao, T.-L.

Cheng, L., see Wang, $\mathrm{X}$.

Cheng, Y.-M., see Pao, T.-L.

Cho, T. H., see Kim, B. H.

Cho, T. H., see Kim, M. S.

Cho, W.-D., see Lee, J.-A.

Cui, L., see Liu, Z.

Dai, M., see Wu, Y.

Dong, Z.-L., see Tian, X.-J.

Ge, S. S., see Guan, F.

Guan, F., Li, L. Y., Ge, S. S. \& Loh, A. P., Robust Human Detection and Identification by Using Stereo and Thermal Images in Human Robot Interaction

Han, D., see Lee, B.

Hou, Z.-G., see Wang, X.

Huang, B., Chen, M., Lee, K. K. \& $\mathrm{Xu}, \mathrm{Y}$., Human Identification

Based on Gait Modeling

\author{
4 (2007) 303 \\ 4 (2007) 347 \\ 4 (2007) 347 \\ 3 (2007) 193 \\ 1 (2007) 39
}

3 (2007) 215

1 (2007) 27

1 (2007) 77

4 (2007) 339

1 (2007) 15

4 (2007) 339

3 (2007) 273

4 (2007) 357

3 (2007) 261

1 (2007) 47

2 (2007) 127

2 (2007) 107

2 (2007) 161

2 (2007) 161

4 (2007) 327

1 (2007) 15

1 (2007) 27
Huang, B., see Chen, M.

Huang, X., see Wang, M.

Jo, K.-H., see Kim, D.-N.

Jo, K.-H., see Kim, T.

Jo, K.-H., see Pham-Ngoc, P.-T.

Kim, B. H., Lee, H. Y. \& Cho, T. H., Fuzzy Rule-Based Enhancing Key Dissemination Method for the Dynamic Filtering-Based Sensor Networks

Kim, D.-N., Trinh, H.-H. \& Jo, K.-H., Object Recognition by Segmented Regions Using Multiple Cues on Outdoor Environment

Kim, J.-H., see Lee, J.-A.

Kim, M. S. \& Cho, T. H., An En-Route Filtering Method in Sensor Networks Using Decision Function

Kim, T. \& Jo, K.-H., Detection of Moving Object Using Remained Background Under Moving Camera

Kim, T.-H., see Pham-Ngoc, P.-T.

Lee, B. \& Han, D., Real-Time Fire and Smoke Detection Using Camera Sequence Image in Tunnel Environment

Lee, D.-W., see Lee, J.-A.

Lee, H. Y., see Kim, B. H.

Lee, J.-A., Lee, D.-W., Kim, J.-H., Cho, W.-D. \& Pajak, J., Adaptive Sensing Schedule Mechanism Considering Neighbor Node in Wireless Sensor Network

Lee, K. K., see Huang, B.

Lee, K. K., see Liang, G.

Li, B. \& Meng, M. Q.-H., A Novel Enhancement Method for Capsule Endoscopy Images
3 (2007) 215

3 (2007) 237

3 (2007) 205

3 (2007) 227

4 (2007) 291

3 (2007) 273

3 (2007) 205

3 (2007) 261

4 (2007) 357

3 (2007) 227

4 (2007) 291

4 (2007) 327

3 (2007) 261

3 (2007) 273

3 (2007) 261

1 (2007) 27

1 (2007) 1

2 (2007) 117 
Li, B. \& Meng, M. Q.-H., Diseases Detection in Wireless Capsule Endoscopy Images with Color Feature

Li, L. Y., see Guan, F.

Li, S., see Chen, W.

Li, Z. \& Liu, P. X., Priority-Based Congestion Control in Multi-Path Wireless Sensor Networks

Liang, G., Lee, K. K. \& Xu, Y., Multi-Resolution Crowd Density Estimation Based on Texture Analysis and Learning from Demonstration

Liang, H., see Chen, W.

Liu, P. X., see Li, Z.

Liu, Q., see Xu, Y.

Liu, Z., Wang, X. \& Cui, L., An Intelligent Soft Measurement Method for Predicting Parameters

Loh, A. P., see Guan, F. Lv, X., see Wang, M. Ma, Y., see Wang, Z.

Mandujano-Valdés, M., see Amaro-Camargo, E. Mei, T., see Chen, W. Meng, M. Q.-H., see Chen, W. Meng, M. Q.-H., see Li, B. Meng, M. Q.-H., see Li, B. Meng, M. Q.-H., see Tong, F.-L. Miao, S., see Chen, W.

Nguyen, Q.-D., see Woo, D.-M.

Pajak, J., see Lee, J.-A.

Pao, T.-L., Cheng, Y.-M., Chen, Y.-T. \& Yeh, J.-H., Performance Evaluation of Different Weighting Schemes on KNN-Based Emotion Recognition In Mandarin Speech

Park, D.-C., see Woo, D.-M.

Pham-Ngoc, P.-T., Kim, T.-H. \& Jo, K.-H., Robust Face Detection for Moving Pictures Under Pose, Rotation, Illumination and Occlusion Changes

Rashidi, A. J., A Novel Decision Fusion Method Based on Multi-Sensor Behavior and Its Application for Networked Target Identification

Reyes-García, C. A., see Amaro-Camargo, E.
3 (2007) 185

3 (2007) 251

2 (2007) 161

1 (2007) 77

4 (2007) 365

1 (2007) 1

1 (2007) 77

4 (2007) 365

3 (2007) 193

1 (2007) 47

2 (2007) 161

3 (2007) 237

1 (2007) 69

4 (2007) 347

1 (2007) 77

1 (2007) 77

2 (2007) 117

3 (2007) 251

2 (2007) 141

1 (2007) 77

4 (2007) 317

3 (2007) 261

4 (2007) 339

4 (2007) 317

4 (2007) 291

4 (2007) 347
Satonaka, T. \& Uchimura, K., Two-Stage Metric Learning Procedure for Facial Signature Authentication

2 (2007) 91

4 (2007) 303

1 (2007) 39

Shen, Y., see Yin, X.-L.

Song, X., Seneviratne, L. D., Althoefer, K. \& Song, Z., Vision-Based Velocity Estimation for Unmanned Ground Vehicles

Song, Z., see Song, X.

Tan, M., see Wang, X.

Tian, X.-J., Wang, Y.-C., Xi, N., Dong, Z.-L. \& Tung, S., Force and Visual Information Acquisition in AFM Based Robotic MWCNT Manipulation

Tong, F.-L. \& Meng, M. Q.-H., Genetic Algorithm Based Visual Localization for a Robot Pet in Home Healthcare System

Trinh, H.-H., see Kim, D.-N.

Tung, S., see Tian, X.-J.

Uchimura, K., see Satonaka, T.

Wang, M., Lv, X. \& Huang, X., Vision Based Motion Control and Trajectory Tracking for Microassembly Robots

Wang, P., see Xu, Y.

Wang, W., Approach of Multiple Moving Targets Detection for Microwave Surveillance Sensors

Wang, X., Hou, Z.-G., Cheng, L., Tan, M. \& Zhu, F., Corridor-Scene Classifying Methods for Mobile Robot Based on Multi-SonarSensor Information Fusion

Wang, X., see Liu, Z.

Wang, Y.-C., see Tian, X.-J.

Wang, Z., Ma, Y. \& Xu, G., A New Approach to Iris Recognition

Woo, D.-M., Park, D.-C. \& Nguyen, Q.-D., Terrain Classification Using $3 D$ Co-Occurrence Features and Neural Networks

4 (2007) 317

Wu, Y. \& Dai, M., Reconstructing the 3D Object with the Rank Constraint of Matrix and Analyzing the Degeneration

Xi, N., see Tian, X.-J.

$\mathrm{Xu}, \mathrm{G}$, see Wang, Z.

1 (2007) 15

1 (2007) 47

2 (2007) 107

1 (2007) 69

Xu, Y., Yu, H., Cai, H., Liu, Q. \& Wang, P., A Novel Integrated
2 (2007) 107

3 (2007) 237

4 (2007) 303

4 (2007) 303

1 (2007) 15

2 (2007) 141

3 (2007) 205

2 (2007) 107

2 (2007) 91

$1(2007) 57$
2 (2007) 127

2 (2007) 107

1 (2007) 69 
Cell-Based Biosensor for

Extracellular Electrophysiological Monitoring

$\mathrm{Xu}, \mathrm{Y}$., see Chen, M.

$\mathrm{Xu}, \mathrm{Y}$., see Huang, B.

$\mathrm{Xu}, \mathrm{Y}$., see Liang, G.

$\mathrm{Xu}, \mathrm{Y}$., see Zhong, Z.

Ye, W., see Zhong, Z.

Yeh, J.-H., see Pao, T.-L.

Yin, X.-L., Chen, H. \& Shen, Y., PBQM: A Priority-Based Queue
3 (2007) 193

3 (2007) 215

1 (2007) 27

1 (2007) 1

4 (2007) 281

4 (2007) 281

4 (2007) 339
Management Method for Wireless Sensor Networks

1 (2007) 39

You, Z., see Chen, W.

1 (2007) 77

$\mathrm{Yu}, \mathrm{H}$., see $\mathrm{Xu}, \mathrm{Y}$.

3 (2007) 193

Zhong, Z., Ye, W. \& Xu, Y.,

Detecting Human Abnormal

Behaviors in Crowd

4 (2007) 281

Zhou, Y., see Chen, W.

1 (2007) 77

Zhu, F., see Wang, $\mathrm{X}$.

1 (2007) 15 